\title{
A NOTE ON THE DICKSON THEOREM ON UNIVERSAL TERNARIES*
}

\author{
BY A. A. ALBERT
}

1. Introduction. A form $f$ with integer coefficients in integer variables is called universal if it represents all positive and negative integers. Evidently, since $f$ is homogeneous, it represents zero for the variables all zero. In case $f=0$ for integral values of the variables not all zero $f$ is called a zero form.

L. E. Dickson $\dagger$ has given a number-theoretic proof of his theorem that every universal ternary quadratic form is a zero form. But his proof is highly technical and consequently quite long and complicated. In the present note I shall give an almost trivial rational proof of Dickson's result. I shall also prove a generalization of his theorem for ternaries over any nonmodular field $F$.

2. Quadratic Forms over $F$. Let $F$ be any non-modular field and let $f\left(x_{1}, \cdots, x_{n}\right)$ be an $n$-ary quadratic form over $F$. Then we shall call $f$ a zero form if $f=0$ for $x_{1}, \cdots, x_{n}$ in $F$ and not all zero. We shall also say that, if every $\rho$ in $F$ is represented by $f$ for $x_{1}, \cdots, x_{n}$ in $F$, the form $f$ is universal over $F$.

It is well known $\ddagger$ that there exists a non-singular linear transformation $x_{i}=\sum a_{i j} X_{j}$ with $a_{i j}$ in $F$ such that

$$
f\left(x_{1}, \cdots, x_{n}\right) \equiv \phi\left(X_{1}, X_{2}, \cdots, X_{n}\right) \equiv \sum_{i=1}^{r} g_{i} X_{i}{ }^{2}+0 \cdot \sum_{j=r+1}^{n} X_{j}{ }^{2},
$$

with $g_{i} \neq 0$ in $F$. The integer $r$ is the rank of $f$. Evidently $f$ is a zero form if and only if $\phi$ is a zero form. But if $r<n$, the form $\phi$ vanishes for any $X_{n}$ in $F$, if $X_{1}=\cdots=X_{r}=0$.

THEOREM 1. Every n-ary of rank $r<n$ is a zero form. Every $n$-ary of rank $n$ is equivalent to

$$
g_{1} X_{1}^{2}+g_{2} X_{2}^{2}+\cdots+g_{n} X_{n}^{2}, \quad\left(g_{i} \text { in } F\right),
$$

with $g_{i}$ all not zero.

* Presented to the Society, April 15, 1933.

$\dagger$ See his Studies in the Theory of Numbers, pp. 17-21.

¥ See Dickson, Modern Algebraic Theories, D. 69 
3. Proof of the Dickson Theorem. Let $f(x, y, z)$ be a universal ternary. By Theorem 1 either $f$ is a zero form of rank less than three or

$$
f(x, y, z) \equiv \phi(X, Y, Z) \equiv \alpha X^{2}+\beta Y^{2}-\gamma Z^{2},
$$

where $\alpha, \beta, \gamma$ are rational, $\alpha \beta \gamma \neq 0$, and $X, Y, Z$ are linearly independent rational linear functions of $x, y, z$. Define

(2) $\delta \equiv \gamma(\alpha \beta)^{-1}, \quad a \equiv \alpha \delta, \quad b \equiv \beta \delta, \quad-a b=-(\alpha \beta \delta) \delta=-\gamma \delta$, so that, for a rational number $\delta \neq 0$,

$$
\delta f \equiv \delta \phi \equiv \psi(X, Y, Z) \equiv a X^{2}+b Y^{2}-a b Z^{2} .
$$

Write $\delta=\delta_{1} \delta_{2}^{-1}$, where $\delta_{1}$ and $\delta_{2}$ are integers. Since $f$ is universal, $f(x, y, z)=\delta_{1} \delta_{2}$ for integer $x, y, z$. Then if $x_{0}=x \delta_{1}^{-1}, y_{0}=y \delta_{1}^{-1}$, $z_{0}=z \delta_{1}^{-1}$, we have $f\left(x_{0}, y_{0}, z_{0}\right)=\delta_{1}^{-2} \delta_{1} \delta_{2}=\delta_{2} \delta_{1}^{-1}=\delta^{-1}$, for rational $x_{0}, y_{0}, z_{0}$. Hence we have proved the following fact.

Lemma 1. If $f$ is universal, $\phi=\delta^{-1}$ for rational $X, Y, Z$.

Let then $\delta^{-1}=\phi, \quad \psi=\delta \phi=\delta \delta^{-1}=1=a X^{2}+b Y^{2}-a b Z^{2}$, and write as a consequence

$$
\xi \equiv 1-a X^{2}=b Y^{2}-a b Z^{2} .
$$

If $\xi=0$, put $\eta=1, \xi=X$, so that

$$
\psi(\xi, \eta, \zeta)=b \cdot 1^{2}-a b \cdot X^{2}=b\left(1-a X^{2}\right)=b \xi=0
$$

for $\eta \neq 0$, and $\phi=\delta^{-1} \psi$ is a zero form. Hence $f$ is a zero form, since $f=0$ for rational $x, y, z$ not all zero if and only if $f=0$ for integers $x, y, z$, not all zero, since $f$ is homogeneous.

Let then $\xi \neq 0$, and put $\eta=a(Z-X Y), \xi=Y-a X Z$, so that

$$
\begin{aligned}
& b \eta^{2}-a b \zeta^{2}= b\left[a^{2}\left(Z^{2}-2 X Y Z+X^{2} Y^{2}\right)\right. \\
&\left.-a\left(Y^{2}-2 a X Y Z+a^{2} X^{2} Z^{2}\right)\right] \\
&=-a b\left[Y^{2}\left(1-a X^{2}\right)-a Z^{2}\left(1-a X^{2}\right)\right] \\
&=-a\left(1-a X^{2}\right)\left(b Y^{2}-a b Z^{2}\right)=-a \xi^{2}, \\
& \delta \phi(\xi, \eta, \zeta) \equiv a \xi^{2}+b \eta^{2}-a b \zeta^{2}=0,
\end{aligned}
$$

and $\phi(\xi, \eta, \zeta)=0$ for $\xi \neq 0$. Hence again $\phi$, and therefore also $f$, are zero forms, and we have proved the Dickson Theorem. The above proof is a rational proof holding for any field $F$ so we have immediately the following result. 
LEMMA 2. If a ternary $f(x, y, z)$ with coefficients in $F$ represents the associated quantity $\delta^{-1}$, then $f$ is a zero form.

4. Universal Ternaries over $F$. We shall now prove the following theorem.

TheOREM 2. A non-singular ternary quadratic form over $F$ is universal over $F$ if and only if it is a zero form.

For let $f$ be a zero form, so that $f(x, y, z)=0$ for $x, y, z$ not all zero and in $F$. Then

$$
\delta \phi \equiv \psi(\xi, \eta, \zeta) \equiv a \xi^{2}+b \eta^{2}-a b \zeta^{2}=0
$$

for $\xi, \eta, \zeta$ not all zero and in $F$. Let $\rho$ be any quantity of $F$, $\sigma=\rho \delta$. If $\xi=0$, then $b\left(\eta^{2}-a \zeta^{2}\right)=0$, whence $\eta^{2}=a \zeta^{2}$, so that $\zeta \eta \neq 0$. Thus write $\xi_{0}=\zeta \eta^{-1}$, from which $a \xi_{0}^{2}=1$. Put

$$
X=0, \quad Y=\frac{\sigma+b^{-1}}{2}, \quad Z=\frac{\sigma-b^{-1}}{2} \xi_{0},
$$

so that, since $1=a \xi_{0}^{2}$,

$$
\begin{aligned}
4 \psi(X, Y, Z) & =b\left[\left(\sigma+b^{-1}\right)^{2}-\left(\sigma-b^{-1}\right)^{2} a \xi_{0}^{2}\right] \\
& =b\left[\left(\sigma+b^{-1}\right)^{2}-\left(\sigma-b^{-1}\right)^{2}\right] \\
& =4 b b^{-1} \sigma=4 \sigma, \quad \text { and } \quad \psi=\sigma .
\end{aligned}
$$

Then $\phi=\delta^{-1} \sigma=\rho$ and hence $f=\rho$ for corresponding $x, y, z$ in $F$.

Next let $\xi \neq 0$. Then $a+b\left(\eta \xi^{-1}\right)^{2}-a b\left(\zeta \xi^{-1}\right)^{2}=0$, and if we write $\eta \xi^{-1}=a \zeta_{0}, \zeta \xi^{-1}=\eta_{0}$, we have $a+a^{2} b \zeta_{0}^{2}-a b \eta_{0}^{2}=0,1=b \eta_{0}^{2}$ $-a b \zeta_{0}^{2}$. Then put

$$
X=\frac{\sigma+a^{-1}}{2}, \quad Y=\frac{\sigma-a^{-1}}{2} a \zeta_{0}, \quad Z=\frac{\sigma-a^{-1}}{2} \eta_{0},
$$

whence

$$
\begin{aligned}
4 \psi(X, Y, Z) & =a\left(\sigma+a^{-1}\right)^{2}+\left(b a^{2} \zeta_{0}^{2}-a b \eta_{0}^{2}\right)\left(\sigma-a^{-1}\right)^{2} \\
& =a\left[\left(\sigma+a^{-1}\right)^{2}-\left(\sigma-a^{-1}\right)^{2}\right]=4 a a^{-1} \sigma=4 \sigma, \\
\psi & =\sigma, \quad \phi=\delta^{-1} \sigma=\rho .
\end{aligned}
$$

Hence in this case also $f=\rho$ as desired, so that $f$ is universal. 
Conversely let $f$ be universal. Then $f$ represents $\delta^{-1}$ and, by Lemma 2, is a zero form. This proves* Theorem 2.

It is well known $\dagger$ that the determinant of the form $\phi(X, Y, Z)$ equivalent to $f$ is $h^{2} d$, where $h$ is the determinant of the transformation. Hence $-\alpha \beta \gamma=h^{2} d$, so that

$$
\delta=\gamma(\alpha \beta)^{-1}=(\alpha \beta \gamma)(\alpha \beta)^{-2}=-d h^{2}(\alpha \beta)^{-2}=-d k^{2},
$$

where $k$ is in $F$. Then

$$
-d f=\delta k^{-2} \phi=k^{-2} \psi(X, Y, Z)=\psi(\xi, \eta, \zeta),
$$

for $X=k \xi \cdot X=k \eta \cdot Z=k \xi$. Hence if $f$ represents the negative of its determinant, the form $\psi=-d f=(-d)^{2}$ represents $d^{2}$, and hence unity, and hence $f$ is a zero form by Lemma 2 . We may therefore replace Lemma 2 by the following statement.

THEOREM 3. If $f$ is a ternary with non-zero determinant $d$, then $-d f(x, y, z) \equiv \psi(X, Y, Z) \equiv a X^{2}+b Y^{2}-a b Z^{2}$ for a suitable transformation. Also $f$ is a universal zero form if and only if $f$ represents $-d$.

In particular the above Theorem 2 holds for the case where $F=R$, the field of all rational numbers. If, however, $a$ is any rational number, then $a=b^{-2} c$, where $b$ and $c$ are integers. Obviously, if $f=a$ for rational $x, y, z$, then $f=c$ for rational $x, y, z$. Hence we have proved a partial converse to Dickson's theorem.

THEOREM 4. A non-singular ternary quadratic form with integer coefficients is a zero form if and only if it represents all integers for rational values of its variables.

The University of Chicago

* It is evident that Theorem 2 is true if it can be proved for forms of type of $\psi(X, Y, Z) \equiv a X^{2}+b Y^{2}-a b Z^{2}$. If $(1, i, j, i j), i^{2}=a, j=b, j i=-i j$, is a generalized quaternion algebra over $F$, then for $a b \neq 0$, this algebra is either a division algebra or a total matric algebra. If $q=X i+Y j+Z i j$, then $q^{2}=\psi(X, Y, Z)$. Hence, if $\psi$ is a zero form, the algebra $Q$ is not a division algebra and there exists a two-rowed matrix whose square is $\sigma$ so that $\psi$ represents $\sigma$. The converse of Theorem 2 is similarly proved. It is in fact this linear algebra theorem (which has long been known to me) which gave me an immediate proof of Theorem 2 as soon as I discovered the reduction given by (1)-(3).

$\dagger$ See Dickson, Modern Algebraic Theories, pp. 64-70. 